\section{THE TEACHING OF ELEMENTARY DYNAMICS}

To THE Editor of ScIEnce: The communication of Professor Wm. Kent in Scrence of December 24, 1915, on the subject heading is particularly interesting as a critical analysis, but the writer does not think Professor Kent's proposed method of teaching the subject is the best way.

As further discussion is invited, a method will now be given, very briefly, that is clear and brief and that beginners readily comprehend.

1. Let a spring-balance be graduated with a set of standard pound weights (metal pieces) at sea level, say at latitude $45^{\circ}$, where $g=32.174 \mathrm{ft}$. per sec. per sec. is the acceleration due to gravity. Now suppose a certain body there, when hung from the spring-balance, to depress the pointer until it reads $W$ pounds; then the pull of the earth at this point on the body is exactly $W$ pounds force.

2. Let the same body be hung from this same spring-balance at any other point where the acceleration of gravity is $g_{1}$ and suppose the pointer reads $W_{1}$ pounds; then the pull of the earth on the body at the second place, is $W_{1}$ pounds force.

3. State as an experimental fact that

$$
W_{1} / g_{1}=W / g \text {. }
$$

This simple equation gives the solution to a number of problems involving weights as measured on the standard spring-balance at different latitudes and altitudes. Give several of these problems.

4. Mass.-Mass of a body means the quantity of matter in the body. It is not supposed to alter in amount by changing the position of the body relative to the earth or to be affected by chemical changes, the expansion or contraction of the body or by any change of the body from a solid to a liquid or gaseous state or a reverse change.

If the body weighs $W$ pounds on the standard spring-balance at the place where the acceleration of gravity is $g \mathrm{ft}$. per sec. per sec., the mass of the body will be assumed to vary with $W / g$, which is likewise unaltered, by eq. (1), by any change of place, volume or condition. If $M$ denote the numerical measure of the mass of the body in question, we can write,

$$
M=k W / g,
$$

where $k$ is a constant for any chosen set of units. For the engineer's system, $k=1$ and,

$$
M=W / g \text {. }
$$

We have now a precise numerical measure of the mass of a body and observe that, at the same place, the mass of a body is directly proportional to its weight. It is not affected by a change of place, by any chemical changes within the body or by any alteration in volume. The student has now a clear-cut, definite idea of the mass of a body and of its measure in the engineer's system. When $W=g, M=1$; hence the unit of mass is the quantity of matter that weighs $g$ lbs. on the spring balance at the place where the acceleration is $g$.

If $W$ is the spring-balance weight at sea level, $45^{\circ}$ latitude, where $g=32.174$, then $M=W / 32.174$ and the unit of mass is the quantity of matter in a body weighing 32.174 lbs. on a spring-balance at sea level, $45^{\circ}$ latitude or $32.174 \mathrm{lbs}$. on a lever balance anywhere.

5. Mass is a fundamental concept and being clearly understood, "density" can be defined, for a homogeneous body, as the ratio $M / V$, where $V$ is the volume of the body of $M$ units of mass.

6. From eq. (2), we have,

$$
W=M g \text {. }
$$

Now if an unbalanced force of $F$ lbs., acting on a body of $M$ units of mass, produces in it an acceleration of $a \mathrm{ft}$. per sec. per sec., the formula giving the relation between $F, M$ and $a$ must reduce to (3) when $F=W, a=g$.

Such a formula is

$$
F=M a \text {. }
$$

This is one of the fundamental formulas of mechanics and the arguments in favor of it should be given as fully as possible, somewhat as in Routh's "Dynamics of a Particle," pp. 18-23 and in connection with Newton's "Three Laws of Motion." The formula is equivalent to the second law, of which the first is a corollary. The formula is readily verified by use of Atwood's machine when $a<g$.

7. From (4), other well-known formulas, $F t=M v, F s=\frac{1}{2} M v^{2}$, etc., can at once be de- 
rived; also by aid of (4) and Newton's third law, that "action and reaction are always equal and contrary" the problem of impact of two particles can be solved.

8. By pursuing the course outlined above, the student has to learn and thoroughly understand, only two simple formulas, $M=W / g$, $F=M a$.

WM. CAIN

Chapel Hill, N. C.

\section{GRAVITATION AND ELECTRICAL ACTION}

IN a paper to be published by the Academy of Science of St. Louis, evidence will be presented which appears to show conclusively, that gravitational attraction is diminished by electrical charges on the acting masses. The suspended masses of the Cavendish experiment are wholly enclosed in a shield of sheet metal. The small observation window is covered with wire gauze. When a knob terminal connected with the influence machine is moved towards or away from a knob terminal connected with the large attracting masses, the suspended masses slowly move to and fro around the vertical line of suspension. No disruptive discharges occur. It is found that gravitational attraction is decreased by either positive or negative electrification. By the to-andfro movement of the knob terminal, the amplitude of vibration can be gradually increased from 2.5 minutes of arc to 50 minutes. It has been established by experimental methods that these results are not due to heat effects.

Francis E. Nipher

\section{THE PRODUCTION OF RADIUM}

To the Editor of ScIEnce: On page 799 of the June 2, 1916, issue of SoIEnce a statement is made in regard to the production of radium by the Standard Chemical Co. in the year 1915, which is not in accord with facts, and I wish to make this correction. The actual amount of radium produced by the Standard Chemical Co. during 1915 was slightly more than 3 grams of radium element and of this the larger proportion was produced in the first three months of the year from radium which was in process of treatment during the latter part of 1914.

In this same article the production of ra- dium at a cost of $\$ 37,599$ per gram by the $\mathrm{Na}$ tional Radium Institute Inc. working in cooperation and under the supervision of the Bureau of Mines, is compared with the market price of radium of $\$ 120,000$ a gram. The radium produced by the National Radium Institute was obtained from high-grade carnotite ore treated without concentration, and the cost of production under these conditions is not properly comparable to the cost of production or the selling price of radium from lower grade ore or concentrates.

Applying the Bureau of Mines process to unconcentrated ore containing about 1.5 per cent. of uranium oxide (which is higher than the average carnotite ore) makes the cost of production nearer $\$ 70,000$ than $\$ 40,000$ per gram. Since this is practically the condition under which commercial producers of radium must operate, it would be fairer to compare cost of production by the Bureau of Mines process on this basis, rather than on the basis of the uncommercial and somewhat artificial conditions, connected with the treatment of the 1,000 tons of high-grade ore. Concentration of the low-grade ore, if practised, naturally reduces the efficiency of extraction, and in this way would raise the cost of production.

While it is true that the war cut off practically the entire European market to radium producers, it must be added that the growing American market for radium has been very adversely influenced by the widespread publishing of statements, from the United States Bureau of Mines, similar to the statement in SCIENCE which we are criticizing. The general effect of these statements has been to lead prospective purchasers of radium to believe that radium would soon be available at enormously reduced prices. Emphasis being laid by the Bureau of Mines on the exceptionally low cost of production, and in general no mention being made of the fact that this low cost of production was in a large measure due to the abnormal and uncommercial conditions under which the Bureau operated.

As regards ore concentration it is also interesting to note that the method used by the Bureau of Mines is one which has been used 